\title{
Relação entre qualidade de vida e tempo de prática paradesportiva em praticantes de Tênis em Cadeira de Rodas
}

\section{Relationship between quality of life and adapted sports practice in wheelchair tennis players}

\author{
Vitor Ciampolini \\ Universidade Federal de Santa Catarina - UFSC - Florianópolis - Brasil \\ vciampolini@gmail.com \\ Larissa Rafaela Galatti \\ Universidade Estadual de Campinas - UNICAMP - Limeira - Brasil \\ lagalatti@hotmail.com \\ Diego Augusto Santos Silva \\ Universidade Federal de Santa Catarina - UFSC - Florianópolis - Brasil \\ diego.augusto@ufsc.br
}

\begin{abstract}
Resumo
A prática paradesportiva tem recebido destaque na promoção de saúde e qualidade de vida para a pessoa com deficiência física. O Tênis em Cadeira de Rodas (TCR) possui papel importante para essa população, entretanto, pouco se sabe sobre a percepção de qualidade de vida de acordo com o tempo de prática da modalidade. O estudo objetivou identificar a associação entre o tempo de prática de TCR e a percepção de qualidade de vida. Participaram do estudo 31 praticantes de TCR com idades entre 19 e 65 anos, estratificados de acordo com o tempo de participação na modalidade: até cinco anos ou mais de cinco anos de prática. A coleta dos dados foi conduzida por meio da aplicação do instrumento WHOQOL-Bref. Os resultados indicam que, de maneira geral, os indivíduos investigados sentem-se satisfeitos com a saúde. Não foi identificada associação significativa entre cada um dos domínios e a percepção total de qualidade de vida de acordo com o tempo de prática na modalidade. Sugere-se que praticantes de TCR não necessitam de um longo período de tempo para perceber a qualidade de vida positivamente. Ademais, aponta-se pelo incentivo de organizações esportivas na promoção do paradesporto para aprimoramento da qualidade de vida de pessoas com deficiência.
\end{abstract}

Palavras-chave: paradesporto, qualidade de vida, tênis em cadeira de rodas.

\section{Abstract}

Adapted sports practice has been highlighted by promoting health and quality of life to people with physical impairments. Wheelchair Tennis (TCR) plays an important role for this population; however, little is known about their quality of life perception according to the time spent in TCR practice. The objective of this study was to identify association between the time spent practicing TCR and the perception of quality of life among people with physical impairments. Participants consisted of 31 TCR players between the ages of 19-65; they were stratified according to their participation time: up to five years or more 
than five years of practice. Data collection was conducted by using the WHOQOL-Bref instrument. The results indicated that, in general, the players investigated feel satisfied with their health. No significant association was found between the groups according to the quality of life domains and the total perception of quality of life analysis. The authors point out that TCR players do not need a long period of time to perceive quality of life positively. In addition, it is highlighted the importance of sports organizations in the promotion of adapted sports for improving people with physical impairments' quality of life.

Keywords: parasports, quality of life, wheelchair tennis.

\section{Introdução}

O termo qualidade de vida refere-se a um constructo multidimensional relacionado à percepção e satisfação da pessoa com as próprias condições de vida, considerando um conjunto de parâmetros individuais e socioambientais (NAHAS, 2010). Para pessoas com deficiência física, a prática paradesportiva se consolida cada vez mais como ferramenta de promoção de saúde e qualidade de vida (GROFF; LUNDBERG; ZABRISKIE, 2009; ZABRISKIE; LUNDBERG; GROFF, 2005; WILHITE; SHANK, 2009), tendo em vista as percepções positivas observadas quando comparados com seus pares fisicamente inativos (NOCE; SIMIM; MELLO, 2009; YAZICIOGLU et al., 2012). Ademais, Laferrier, Teodorski e Cooper (2015) identificaram que indivíduos que praticam o paradesporto por um maior período de tempo apresentam maiores escores de qualidade de vida em comparação com indivíduos que praticam há menos tempo. Estes achados contribuem no fomento da integração de pessoas com deficiência no contexto de prática paradesportiva, bem como na manutenção do praticante já inserido neste.

Tendo em vista as diversas modalidades paradesportivas desenvolvidas para a prática da pessoa com deficiência física, o Tênis em Cadeira de Rodas, especificamente, tem apresentado destaque devido a gama de experiências positivas que pode proporcionar aos praticantes, tanto para 0 esporte de representação, quanto para 0 esporte relacionado à saúde (CIAMPOLINI et al., 2014). Entretanto, ainda não se sabe na realidade brasileira se indivíduos que praticam Tênis em Cadeira de Rodas por mais tempo apresentam maiores escores de percepção de qualidade de vida em comparação aos indivíduos que praticam há menos tempo. Portanto, este estudo busca identificar a associação entre o tempo de prática de Tênis em Cadeira de Rodas e a percepção de qualidade de vida.

\section{Métodos}

31 praticantes brasileiros de Tênis em Cadeira de Rodas (25 homens e seis mulheres) com idades entre 19 e 65 anos (Média $=39,2$; DP $=11,5$ ) participaram do estudo. Para análise da qualidade de vida de acordo com o tempo de prática de Tênis em Cadeira de Rodas, os participantes foram estratificados em dois grupos: até cinco anos de prática (13 indivíduos) e mais de cinco anos de prática (18 indivíduos).

Os dados foram coletados em dois momentos: em Maio de 2014, por meio de uma visita a um projeto de extensão de uma universidade do sul do Brasil e, em Outubro de 2014, por meio do convite aos participantes de um torneio internacional de Tênis em Cadeira de Rodas realizado no sul do Brasil.

Para coleta dos dados utilizou-se o instrumento WHOQOL-bref, proposto por WHOQOL Group (1998) e traduzido e validado para o Português brasileiro por Fleck et al. (2000). O instrumento avalia a autopercepção de qualidade de vida por meio de 26 questões, as quais apresentam cinco opções de resposta por meio de uma escala Likert. 
Além disso, ressalta-se que o instrumento é dividido em quatro domínios de análise: físico, psicológico, social e ambiente.

Para tabulação dos dados utilizou-se o software Microsoft Office Excel (versão 2010). Já para a análise dos dados utilizou-se estatística descritiva com valores de média, desvio padrão e porcentagem. Para as variáveis categóricas empregou-se o teste Exato de Fisher. Para verificar a normalidade dos dados das variáveis quantitativas empregouse o teste de Shapiro-Wilk. Considerando que os domínios psicológico e social não apresentaram distribuição normal, empregou-se a estatística inferencial não-paramétrica. Para a comparação dos grupos em relação ao tempo de prática de Tênis em Cadeira de Rodas utilizou-se o teste $\mathrm{T}$ de Student para amostras independentes ou o equivalente não-paramétrico (teste de Mann-Whitney $U$ ). Todas as análises foram realizadas no software SPSS (versão 23.0) com nível de significância estabelecido em 5\%.

Salienta-se que esta pesquisa foi aprovada pelo Comitê de Ética em Pesquisa com Seres Humanos da UFSC (CEPSH) por meio do parecer 539.112 e para participar do estudo os sujeitos assinaram o Termo de Consentimento Livre e Esclarecido (TCLE).

\section{Resultados}

Os achados indicam que, de maneira geral, os praticantes de Tênis em Cadeira de Rodas investigados sentem-se satisfeitos com a saúde $(n=23)$, visto que $74,2 \%$ dos indivíduos apresentaram tal percepção. Quando estratificados em grupos de acordo com o tempo de prática, observou-se que o grupo com mais tempo de prática apresenta maior porcentagem de indivíduos satisfeitos com a saúde com relação ao grupo com menos prática, sendo $77,8 \%$ e $69,2 \%$, respectivamente. Entretanto, não se identificou diferença significativa entre as variáveis investigadas $(p>0,05)$. A tabela 1 apresenta os achados mencionados acima.

Tabela 1: Percepção geral de saúde de acordo com o tempo de prática

\begin{tabular}{lccc}
\hline \multirow{2}{*}{ Grupos } & \multicolumn{2}{c}{ Satisfação com a Saúde } & \multirow{2}{*}{ p-valor } \\
\cline { 2 - 3 } & Satisfeito & Insatisfeito & \\
\hline Tempo de Prática & $\mathrm{n}(\%)$ & $\mathrm{n}(\%)$ & \\
Até 5 anos & $09(69,2)$ & $04(30,8)$ & \\
Mais de 5 anos & $14(77,8)$ & $04(22,2)$ & \\
Total & $23(74,2)$ & $08(25,8)$ & \\
\hline a - Teste Exato de Fisher & & \\
Fonte: Autoria própria $(2016)$. &
\end{tabular}

Do mesmo modo, identificou-se que no grupo de praticantes de Tênis em Cadeira de Rodas investigados, a percepção total de qualidade de vida não apresentou associação significativa com o tempo de prática da modalidade $(p>0,05)$, considerando indivíduos com até cinco anos de prática e indivíduos com mais de cinco anos de prática. Além disso, nenhum dos quatro domínios abrangidos pelo instrumento WHOQOL-Bref apresentou associação significativa com o tempo de prática $(p>0,05)$. Entretanto, observou-se maiores escores em todas as médias das variáveis investigadas no grupo praticante há mais de cinco anos, o que pode sugerir certa tendência de percepção mais positiva em comparação com o grupo praticante há menos de cinco anos. Estes dados podem ser observados na Tabela 2.

Tabela 2: Análise da qualidade de vida de tenistas em cadeira de rodas com relação ao tempo de prática na modalidade.

\begin{tabular}{lccc}
\hline \multirow{2}{*}{ Domínios } & \multicolumn{2}{c}{ Tempo de Prática } & \multirow{2}{*}{ p-valor } \\
\cline { 2 - 3 } & Até 5 anos & Mais de 5 anos & \\
\hline \multirow{2}{*}{ Físico } & Média \pm DP & Média \pm DP & \\
\hline
\end{tabular}




\begin{tabular}{lccc}
\hline Psicológico & $78,53 \pm 13,27$ & $81,71 \pm 9,39$ & $0,657^{\mathrm{c}}$ \\
Social & $78,85 \pm 12,55$ & $79,63 \pm 14,63$ & $0,743^{\mathrm{c}}$ \\
Ambiente & $70,67 \pm 15,33$ & $71,53 \pm 12,07$ & $0,863^{\mathrm{b}}$ \\
Total & $73,84 \pm 11,30$ & $77,02 \pm 8,85$ & $0,387^{\mathrm{b}}$ \\
\hline b - Teste T de Student & & \\
c- Teste de Mann-Whitney U & & \\
\multicolumn{2}{l}{ Fonte: Autoria própria (2016). } & &
\end{tabular}

\section{Conclusão}

Os achados indicam primeiramente que a maioria dos praticantes de Tênis em Cadeira de Rodas investigados sentem-se satisfeitos com a saúde independente do tempo de prática da modalidade. Da mesma maneira, identificou-se que o tempo de prática de Tênis em Cadeira de Rodas não está associado com nenhum dos quatro domínios de qualidade de vida investigados, nem com a percepção total de qualidade de vida.

Contudo, apesar de estas variáveis não terem apresentado relação significativa, identificou-se médias superiores nos domínios de qualidade de vida investigados no grupo com maior tempo de prática, bem como na percepção total de qualidade de vida. Estes achados podem sugerir certa tendência de melhores percepções de qualidade de vida por meio de um maior período de prática.

Sugere-se que praticantes de Tênis em Cadeira de Rodas não necessitam de um longo período de tempo de prática para perceberem significativamente melhor a qualidade de vida, visto que pessoas com deficiência praticantes desta modalidade já apresentam percepção positiva de qualidade de vida e satisfação com a saúde independente do tempo de prática. Deste modo, aponta-se pela necessidade de entidades esportivas, dirigentes e familiares promoverem e incentivarem a participação de pessoas com deficiência no contexto paradesportivo, de modo a contribuir no aprimoramento da qualidade de vida desta população.

\section{Referências}

CIAMPOLINI, V. et al. Benefícios Psicossociais do Treinamento Esportivo em Tênis em Cadeira de Rodas. ConScientiae Saúde, v. 13, Suplemento, p. 119-122, 2014.

FLECK, M. P. et al. Aplicação da versão em português do instrumento abreviado de avaliação da qualidade de vida "WHOQOL-bref". Revista de Saúde Pública, v. 34, n. 2, p. 178-183, 2000.

GROFF, D. G.; LUNDBERG, N. R.; ZABRISKIE, R. B. Influence of adapted sport on quality of life: Perceptions of athletes with cerebral palsy. Disability and Rehabilitation, $v$. 31, n. 4, p. 318-326, 2009.

LAFERRIER, J. Z.; TEODORSKI, E.; COOPER, R. A. Investigation of the impact of sports, exercise, and recreation participation on psychosocial outcomes in a population of veterans with disabilities: a cross-sectional study. American Journal of Physical Medicine and Rehabilitation, v. 94, n. 12, p. 1026-1034, 2015.

NAHAS, M. V. Atividade Física, Saúde e Qualidade de Vida: conceitos e sugestões para um estilo de vida ativo. $4^{\circ}$ Edição, Londrina: Midiograf, 2010. 318 p. 
NOCE, F.; SIMIM, M. A. D. M.; MELLO, M. T. D. A percepção de qualidade de vida de pessoas portadoras de deficiência física pode ser influenciada pela prática de atividade física?. Revista Brasileira de Medicina do Esporte, v. 15, n. 3, p. 174-178, 2009.

WILHITE, B.; SHANK, J. In praise of sport: Promoting sport participation as a mechanism of health among persons with a disability. Disability and Health Journal, v. 2, n. 3, p. 116-127, 2009.

WHOQOL GROUP. Development of the World Health Organization WHOQOL-BREF quality of life assessment. Psychological Medicine, v. 28, n. 3, p. 551-558, 1998.

YAZICIOGLU, K. et al. Influence of adapted sports on quality of life and life satisfaction in sport participants and non-sport participants with physical disabilities. Disability and Health Journal, v. 5, n. 4, p. 249-253, 2012.

ZABRISKIE, R. B.; LUNDBERG, N. R.; GROFF, D. G. Quality of life and identity: The benefits of a community-based therapeutic recreation and adaptive sports program. Therapeutic Recreation Journal, v. 39, n. 3, p. 176, 2005. 